\section{Breite Diagnostik zum kleinen Preis}

$\mathrm{M}$ it den in-vitro-Testpanels (CLAAllergen-Panels) der Firma ADL ist es möglich, auf 20 verschiedene Allergene zu testen. Das System, das auf einer Kopplung der Allergene an eine solid phase (Zellulosefäden) basiert, existiert in drei verschiedenen $\mathrm{Zu}$ sammensetzungen, so dass neben einem primären Allergiescreening auch ein spezieller Test auf Inhalationsallergene („Inhalations-Panel“) bzw. Nahrungsmittelallergene („Nahrungsmittelpanel“) möglich ist (s. Kasten).

L. Klimek, Wiesbaden, führte im Rahmen einer Evaluationsstudie mit den CLA-Panels mehr als 4.000 Allergennachweise durch. Dabei fand er für Inhalationsallergene eine Sensitivität von $90,2 \%$ und eine Spezifität von 92,1\%, gemessen an den Ergebnissen von Hauttests, Klinik/Anamnese und Provokationsstests. Diese Ergebnisse sind mit denjenigen vergleichbar, die sich mit RAST und ELISA zum Nachweis spezifischer IgE-Antikörper erzielen lassen. Der große Vorteil der CLAPanels: Mit ihnen lässt sich das Allergiescreening wesentlich kostengünstiger durchführen. So kostet die Untersuchung, mit der 20 Einzelallergene erfasst werden, nur 65 DM. Bei einer klassischen „RAST“-Einzelallergenanforderung kostet die Bestimmung eines Allergens im O-III-Labor schon 15
DM, womit die Paneldiagnostik bereits bei einer Anforderung von vier oder mehr Einzelallergenen die kostengünstigere und informativere Alternative ist.

Nach Informationen von Matritech
CLA-Allergen-Panels: Dreiteilung der Allergene für die Diagnostik

\begin{tabular}{|c|c|c|}
\hline Inhalations-Panel & Nahrungsmittel-Panel & Atopie-Panel \\
\hline _Erle & — Krabbe & — Karotte \\
\hline _- Hasel & _ Dorsch & _ Apfel \\
\hline — Birke & _ Karotte & — Kartoffel \\
\hline — Beifuß & —Apfel & _Erdnuss \\
\hline — Lieschgras & — Kartoffel & —Soja \\
\hline —Roggen & _ Tomate & — Reis \\
\hline —Wegerich & —Sellerie & — Weizen \\
\hline _ Federmischung & _Pfirsich & _ Dorsch \\
\hline _- Hamster & _Walnuss & _ Milch \\
\hline — Meerschweinchen & _Erdnuss & _ Eiklar \\
\hline _Kaninchen & _ Haselnuss & _Birke \\
\hline — Katze & _ Mandel & — Beifuß \\
\hline — Hund & —Soja & — Lieschgras \\
\hline — Pferd & — Roggenmehl & — Katze \\
\hline — Penicillium & —Weizenmehl & _ Hund \\
\hline notatum & — Sesam & — Pferd \\
\hline — Alternaria tenuis & _ Kasein & _Cladosporium \\
\hline —Cladosporium & _ Milch & _ Aspergillus \\
\hline — Aspergillus & _Eiklar & — Dermat. farinae \\
\hline — Dermat. farinae & — Eigelb & _ Dermat. pteronyss. \\
\hline
\end{tabular}

\section{Adrenalin-Injektor: Retter in der Not}

\footnotetext{
$\mathrm{M}$ it den wärmeren Monaten beginnt auch die Saison für Bienen und Wespen - und damit die risikoreiche Zeit für Patienten mit schweren Insektengift-Allergien: Innerhalb von Minuten nach einem Stich kann sich ein lebensbedrohlicher anaphylaktischer Schock entwickeln, der bereits in der Frühphase einer sofortigen effektiven medikamentösen Behandlung bedarf. Experten fordern daher, Risikopatienten für den Ernstfall mit einer Notfallapotheke zur Selbstmedikation zu wappnen. Diese sollte ein Antihistaminikum, ein Glukokortikoid sowie ein schnell wirksames und sicher zu applizierendes Adrenalin-Präparat enthalten.
}

In Deutschland werden pro Jahr bis zu 40 Todesfälle als Folge eines anaphylaktischen Schocks nach Insektenstich registriert. Die anaphylaktischen Reaktionen beginnen sehr rasch, etwa bei $50 \%$ aller Patienten innerhalb weniger Minuten bis zu einer halben Stunde.

Bereits bei ersten klinischen Zeichen einer drohenden Anaphylaxie wird die sofortige Gabe von Adrenalin (Epinephrin) unumgänglich. Seit dem Inkrafttreten der FCKW-Halon-Verbotsordnung sind in Deutschland die früher für diesen Zweck gerne eingesetzten Adrenalin-Dosieraerosole nur unter besonderen Bedingungen erhältlich. Fastjekt ${ }^{\circledR}$, ein automatischer Adrenalin-Injektor zur i.m.-Applikation, gilt heute in Fachkreisen als alternatives Mittel der Wahl für die notfallmäßige Adrenalingabe im Rahmen der Laien-Selbsthilfe. Diese in Deutschland derzeit einzige für die Notfallversorgung des anaphylaktischen Schocks zugelassene Adrenalin-Fertigspritze zeichnet sich durch eine rasche, sichere, Patienten- und Situations-gerechte Handhabbarkeit sowie eine schnell einsetzende Adrenalinwirkung innerhalb von wenigen Minuten aus. Dank automatischer Injektion ist auch unter schwierigen Notfallbedingungen die Verabreichung einer definierten Wirkstoffmenge in den Muskel gewährleistet und damit die entscheidende Voraussetzung für einen rechtzeitigen Wirkeintritt und erfolgreichen Einsatz im Ernstfall geschaffen.

Nach Informationen von Allergopharma 